\title{
Effect of training traditional birth attendants on neonatal mortality (Lufwanyama Neonatal Survival Project): randomised controlled study
}

An incorrect numerator was used to calculate the rate ratio of neonatal deaths from serious infection in this paper by

Christopher J Gill and colleagues (BMJ 2011;342:373, doi:10. 1136/bmj.d346). The values in table 4 are correct, but two study deaths occurring outside the neonatal period (that is, after 27 days) had been included in the intervention group. This meant that the cluster adjusted rate ratios (shown in the text but not in the table) included 18 deaths from serious infection [not 16, as shown in the table]. The correct risk ratio is $0.73(95 \%$ confidence interval 0.36 to 1.51$)$ [not 0.82 (0.41 to 1.63$)$ ]. The conclusions of the paper are unaffected

Cite this as: BMJ 2011;342:d2301 